\title{
Working Memory for Conjunctions Relies on the Medial Temporal Lobe
}

\author{
Ingrid R. Olson, ${ }^{1}$ Katie Page, ${ }^{3}$ Katherine Sledge Moore, ${ }^{1}$ Anjan Chatterjee, ${ }^{1,2}$ and Mieke Verfaellie ${ }^{3}$ \\ ${ }^{1}$ The Center for Cognitive Neuroscience, University of Pennsylvania and ${ }^{2}$ Department of Neurology, University of Pennsylvania Medical School, \\ Philadelphia, PA 19104, and ${ }^{3}$ Boston University School of Medicine and VA Boston Healthcare System, Boston, MA 02130
}

A prominent theory of hippocampal function proposes that the hippocampus is importantly involved in relating or binding together separate pieces of information to form an episodic representation. This hypothesis has only been applied to studies of long-term memory because the paradigmatic view of the hippocampus is that it is not critical for short-term forms of memory. However, relational processing is important in many working memory tasks, especially tasks using visual stimuli. Here, we test the hypothesis that the medial temporal lobes are important for relational memory even over short delays. The task required patients with medial temporal lobe amnesia and controls to remember three objects, locations, or object-location conjunctions over 1 or $8 \mathrm{~s}$ delays. The results show that working memory for objects and locations was at normal levels, but that memory for conjunctions was severely impaired at $8 \mathrm{~s}$ delays. Additional analyses suggest that the hippocampus per se is critical for accurate conjunction working memory. We propose that the hippocampus is critically involved in memory for conjunctions at both short and long delays.

Key words: hippocampus; associative; amnesia; visual; memory; long-term; short-term; chunking; binding

\section{Introduction}

Bilateral damage to the hippocampus and related medial temporal lobe structures causes severe memory impairments (Scoville and Milner, 1957). An important question in memory research is how best to characterize the role of the medial temporal lobes (MTLs) in memory. The relational-processing theory proposes that the MTL system is important for encoding into memory what has been described as contextual (Hirsh, 1974), configural (Sutherland and Rudy, 1989), or relational (Cohen and Eichenbaum, 1993) information. The underlying theme of these proposals is that the MTL is important for binding together different elements of a memory trace (Eichenbaum, 1999).

Several lines of research support this hypothesis. MTL amnesics have profound difficulties in remembering learned word pairs as compared with single words (Cermak, 1976; Winocur and Weiskrantz, 1976; Giovanello et al., 2003; Turriziani et al., 2004). Several neuroimaging studies have reported greater activations in MTL structures during encoding (Henke et al., 1997; Davachi et al., 2003; Kirwan and Stark, 2004; Ranganath et al., 2004) and retrieval (Yonelinas et al., 2001; Giovanello et al., 2004) of the relationship between stimuli as opposed to separate stimuli.

The question we address in this paper is whether the MTL is

Received May 13, 2005; revised Jan. 10, 2006; accepted March 22, 2006.

This work was supported by National Institute of Mental Health Grants R01 MH071615-01 (I.0.) and R01 MH057681 (M.V.), and by the Medical Research Service of the Department of Veterans Affairs (M.V.). We thank Michael Tarr and Bruno Rossion for providing stimuli, Marianna Stark and Youssef Ezzyat for helping with collection of control data, and Michael Kahana and Charan Ranganath for helpful discussions.

Correspondence should be addressed to Ingrid R. Olson, Center for Cognitive Neuroscience, University of Pennsylvania, 3720 Walnut Street, Room B51, Philadelphia, PA 19104. E-mail: iolson@psych.upenn.edu.

DOI:10.1523/JNEUROSCI.1923-05.2006

Copyright $\odot 2006$ Society for Neuroscience $\quad$ 0270-6474/06/264596-06\$15.00/0 involved in binding processes in long-term memory (LTM) only, or whether it is more generally involved in mnemonic binding, regardless of time that intervenes between study and test. Studies to date have primarily examined LTM for conjunctions because of the fact that a great deal of evidence suggests that MTL damage leads to delay-dependent memory loss. Whereas working memory (WM) ${ }^{a}$ for digits (Wickelgren, 1968; Cave and Squire, 1992), tones (Wickelgren, 1968), words (Baddeley and Warrington, 1970), or single-dot locations (Cave and Squire, 1992) appears to be spared in MTL amnesia, LTM for most types of information is impaired (Cohen and Eichenbaum, 1993).

Although simple forms of WM may not be affected by MTL damage, it is possible that forms of WM that require relational processing rely on the MTL. There is mounting evidence that relational memory is quite different from simpler forms of memory (Mitchell et al., 2000a,b), thus the presence of intact memory for simple features provides no information about whether or not memory for the relationship between stimuli is intact. Also, one neuroimaging study showed that the hippocampus is activated in relational memory tasks when delays are as short as $8 \mathrm{~s}$ (Mitchell et al., 2000a). Because neuroimaging is a correlational procedure, such evidence alone cannot tell us whether the MTL is necessary for accurate performance on WM tasks.

Here, we test MTL amnesics and controls in two experiments that required them to remember either three sequentially pre-

\footnotetext{
aThe terms "short-term memory" (STM) and "working memory" (WM) refer to different things, with the forme emphasizing the storage aspect of memory and the latter emphasizing both the storage and the manipulation of information held in memory. Although the distinction between storage and manipulation is of theoretical interest, in practice, the terms STM and WM have been used somewhat interchangeably. For instance, visual memory tasks with short delays between two arrays are sometimes referred to as "visual working memory" and sometimes referred to as "visual short-term memory." In this paper we have decided that "visual working memory" is a more neutral and ubiquitous term to use for this study.
} 


\begin{tabular}{|c|c|c|c|c|c|c|c|c|c|}
\hline \multirow[b]{2}{*}{ Age } & \multirow[b]{2}{*}{ Sex } & \multirow[b]{2}{*}{ Edu } & \multirow[b]{2}{*}{ Etiology } & \multirow[b]{2}{*}{ MTL Lesion } & \multirow{2}{*}{$\frac{\text { WAIS-III }}{\text { VIQ }}$} & \multicolumn{4}{|c|}{ WMS-III } \\
\hline & & & & & & GM & $A D$ & VD & WM \\
\hline & & & & Description & & & & & \\
\hline $48^{*}$ & M & 14 & Anoxia & $\mathrm{BiH}$ & 111 & 59 & 52 & 72 & 96 \\
\hline 76 & $\mathrm{~F}$ & 12 & Anoxia & $\mathrm{BiH}$ & 107 & 59 & 64 & 65 & 83 \\
\hline $52^{*}$ & M & 17 & Anoxia & Pacemaker & 134 & 70 & 67 & 75 & 88 \\
\hline 75 & M & 18 & Anoxia & $\mathrm{BiH}$ & 113 & 75 & 80 & 72 & 102 \\
\hline $54^{*}$ & $\mathrm{~F}$ & 12 & Anoxia & Bi H, PRC, R amg & 83 & 52 & 55 & 56 & 91 \\
\hline $46^{*}$ & $\mathrm{~F}$ & 14 & Anoxia & $\mathrm{BiH}$ & 90 & 45 & 52 & 53 & 93 \\
\hline $49^{*}$ & M & 14 & Enceph & Bi H, PRC, ERC, amg & 92 & 45 & 55 & 56 & 85 \\
\hline 61 & $\mathrm{~F}$ & 12 & Enceph & Bi H, PRC, ERC, PHG, R amg & 106 & 69 & 77 & 68 & 111 \\
\hline $76^{*}$ & $\mathrm{~F}$ & 18 & Enceph & Bi H, PRC, ERC, amg, temporal pole & 135 & 45 & 58 & 53 & 141 \\
\hline $42^{* *}$ & M & 16 & Anoxia & Bi H, PRC, ERC, L fusiform & 86 & 49 & 52 & 53 & 93 \\
\hline
\end{tabular}

Edu, Education in years; VIQ, verbal intelligence quotient; GM, general memory index; $A D$, auditory delay index; $V D$, visual delay index; WM, working memory index; Enceph, encephalitis; Bi, bilateral; $H$, hippocampus; PRC, perirhinal cortex; ERC, entorhinal cortex; amg, amygdala; PHG, parahippocampal gyrus; R, right; L, left; WAIS-III, Wechsler Adult Intelligence Scale, third edition; WMS-III, Wechsler Memory Scale, third edition. *Patients tested in both experiments. **Patient tested only in experiment 2 .

sented objects, locations, or object plus location conjunctions (Mitchell et al., 2000a,b). If the MTL is important for relational binding at short delays, then amnesic patients should have intact memory for objects or locations, but impaired memory for objects plus locations. Alternatively, if the long-term binding hypothesis is true, amnesics should show intact performance in all conditions.

\section{Materials and Methods}

Participants. The lesion group in experiment 1 consisted of nine patients with bilateral medial temporal lobe damage (for details, see Table 1). Amnesia resulted from an anoxic episode $(n=6)$ or from encephalitis $(n=3)$. Inspection of magnetic resonance images (MRIs) showed that four of the patients had damage limited to the hippocampus, and four of the patients had damage extending into other MTL structures including the perirhinal and entorhinal cortex. MRI data were not available for one of the anoxic patients because of the presence of a pacemaker. The average verbal intelligence quotient (IQ) as measured by the Wechsler Adult Intelligence Scale, third edition (Wechsler, 1997a), was 108. The general-memory score on the Wechsler Memory Scale, third edition (Wechsler, 1997b), was 58 and the delayed-memory score was 63 . Six of these patients and one new patient were tested 3-6 months later in experiment 2.

Control participants in experiment 1 were 10 healthy adults (five males, five females, 45-77 years, $M=64$ years) with an average of 14 years of education and an average verbal IQ of 113. Control participants in experiment 2 were eight healthy adults (two males, six females) with an average of 14 years of education and an average verbal IQ of 113. There was no difference between the MTL group and the respective control group in terms of age, education, or verbal IQ in either experiment (all $p$ values $>0.10$ ). All participants were cooperative and attentive and had normal or corrected-to-normal visual acuity.

Design and statistical analysis. In addition to the betweensubject variable (patient vs control), there were two withinsubject variables: (1) condition (object, location, or object plus location conjunction, referred to hereafter as "conjunction"), and (2) interstimulus interval (ISI; 1 or $8 \mathrm{~s}$ ). Data from object and location trials were combined into a "feature" condition after assuring that there was no accuracy difference between these conditions (Mitchell et al., 2000b). This resulted in a $2 \times 2 \times 2$ design.

Hit rates (responding "yes" on a "match" trial) and false alarm rates (responding "yes" on a "nonmatch" trial) were used to calculate corrected recognition (hits minus false alarms).
Materials. The stimuli consisted of 259 colorized Snodgrass and Vanderwart-like renderings (Rossion and Pourtois, 2004) of common objects and animals, which were presented within a $3 \times$ 3 black grid on a white background. Stimulus size was $\sim 5 \mathrm{~cm}$ by $5 \mathrm{~cm}$. The $3 \times 3$ grid was $20 \mathrm{~cm}$ by $20 \mathrm{~cm}$. Stimuli were shown without replication.

Equipment. Participants were tested individually on either a laptop computer or a desktop computer. They sat at an unrestricted viewing distance of $\sim 57 \mathrm{~cm}$, at which distance $1 \mathrm{~cm}$ corresponds to $1^{\circ}$ viewing angle. The experiment was programmed in E-prime.

Task and procedure. The task was to remember either three objects, locations, or object-location conjunctions over a short delay period and then make a recognition judgment. Trial onset was signaled by the onset of the grid, followed by the sequential presentation of 3 stimuli. Each stimulus was presented for $1 \mathrm{~s}$, and separated by a $13 \mathrm{~ms}$ ISI. Each stimulus appeared within one of nine randomly chosen locations, with the restriction that there was no repetition of locations within a three-item sequence. After the memory sequence finished, there was an unfilled delay interval of 1 or $8 \mathrm{~s}$. Last, the probe image was shown and a recognition judgment was elicited. Participants pressed one key for a "same" response and a second key for a "different" response. After the response was entered, the screen cleared and there was a $500 \mathrm{~ms}$ blank intertrial interval.

An equal number of match and nonmatch trials were randomly intermixed in each condition. Probes on match trials were sampled from all items/locations shown on the memory image. Depending on the condition, participants were asked to indicate whether the object, location, or conjunction of object and location, matched one seen in the memory image. The probe image during the "object" block of trials consisted of one drawing presented in the center of the grid. On match trials, the drawing had been presented in the memory sequence for that trial, whereas on nonmatch trials, a novel stimulus was presented. The probe image during the "location" block of trials consisted of one filled black circle that appeared in one of the nine grid locations. On match trials, the black circle appeared in one of the previously filled locations. On nonmatch trials, it appeared in a location that had not been filled during the memory sequence. The probe image during the "conjunction" trials consisted of one drawing that appeared in one of the nine grid locations. On match trials, both the object and the location matched the object plus location conjunction from the memory sequence. On nonmatch trials, an object from the memory sequence for that trial was recombined 
with an incorrect location from that trial. By using recombined object and location information, subjects were forced to consider both pieces of information to make their answer. In experiment 1 , each of the three conditions was administered in a separate block of six practice trials and 36 experimental trials, with the order of conditions counterbalanced across subjects (Fig. 1). In experiment 2, for reasons explained below, object and location trials were intermixed in one block of six practice trials and 72 experimental trials, and conjunction trials were administered in a separate block of six practice trials and 36 experimental trials. The order of blocks was again counterbalanced across subjects. Participants took breaks at the end of each block to rest and to receive a new set of instructions for the subsequent block of trials. Accuracy, not speed, was emphasized.

\section{Results}

\section{Experiment 1}

Figure $2 a$ shows the results of our analysis. A repeated-measures ANOVA with condition, delay, and group as factors found a main effect of condition $\left(F_{(1,17)}=19.49 ; p<0.0004\right)$ with lower overall accuracy when conjunctions had to be remembered $(M=$ $68 \%)$ compared with objects or locations $(M=91 \%)$. In contrast, a longer delay interval did not generally decrease performance $\left(F_{(1,17)}=2.96 ; p=0.10\right)$. The effect of group membership was marginally significant $\left(F_{(1,17)}=3.75 ; p=0.07\right)$, a finding that is more readily understood when the interactions are examined. Of interest, the interaction of condition by delay by group was significant $\left(F_{(1,17)}=6.80 ; p<0.02\right)$. This was because of similar levels of performance by amnesics and controls in the feature condition $(M=88$ vs $98 \%)$ and conjunction condition at the $1 \mathrm{~s}$ delay $(M=73$ vs $73 \%)$, and similar performance levels in the feature condition at the 8 s delay ( $M=88$ vs $88 \%)$ but dramatically lower performance by amnesics in the conjunction condition at the $8 \mathrm{~s}$ delay $(M=45 \mathrm{vs} 79 \%)$. None of the other interactions were significant (all $p$ values $>0.15$ ).

To assess whether binding deficits found in experiment 1 were caused by hippocampal or extra-hippocampal damage in the MTL, we compared the performance of patients with damage limited to the hippocampus (termed " $\mathrm{H}$ " group; $n=4$ ) to that of patients with more extensive MTL damage (termed " $\mathrm{H}+$ " group; $n=4)$ on the conjunction trials. No difference was found between groups $\left(F_{(1,6)}=1.97 ; p=0.21\right)$ and the interaction of group with delay was not significant $(F<1)$. Of interest, the groups did not differ at the 8 s delay $(\mathrm{H}+$ group: $M=62 \%, \mathrm{SE}=$ $21 \%$; H group: $M=40 \%, \mathrm{SE}=19 \%$; $p=0.47$ ).

These findings are consistent with our hypothesis that the hippocampus is important for relational processing even over brief delays. However, one explanation for the results of experiment 1 that cannot be ruled out is that more information had to be remembered on conjunction trials as compared with feature trials. Were the deficits experienced by amnesics in experiment 1 caused by a binding problem, as we hypothesized, or were they caused by the greater memory load in the conjunction condition than in the feature condition? If the latter hypothesis is true,

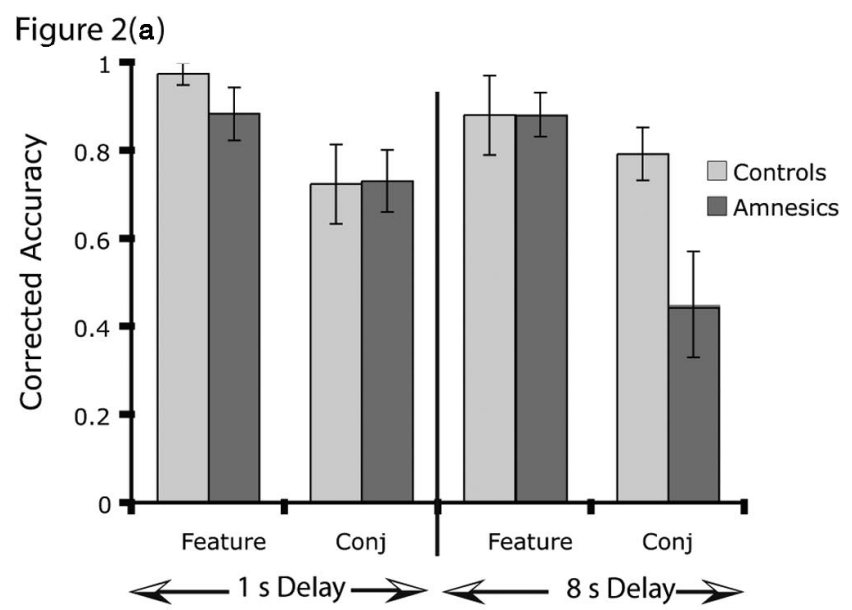

Figure 2(b)

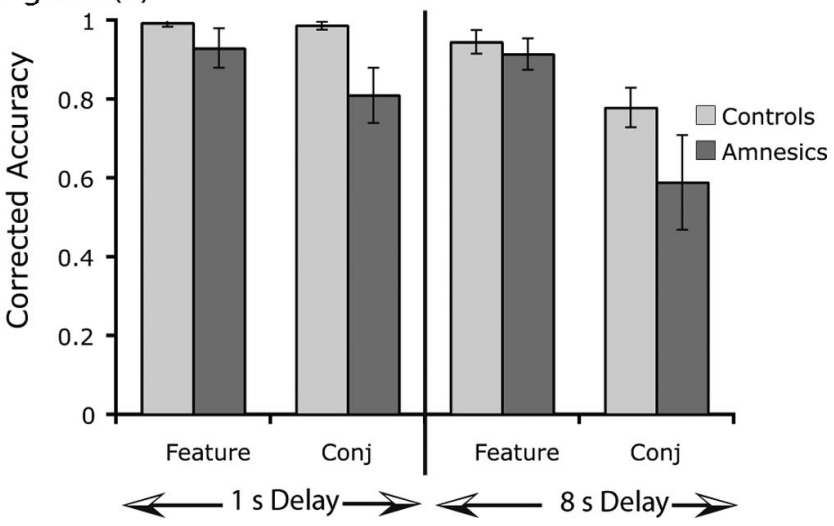

Figure 2. Corrected recognition (hit rate-false alarm rate) as a function of feature or conjunction condition for the 1 and $8 \mathrm{~s}$ ISIs for Experiment $1(\boldsymbol{a})$ and Experiment $2(\boldsymbol{b})$. Error bars represent SEM.

amnesics should perform as poorly on feature trials as on conjunction trials when the memory load is equated.

In experiment 2, MTL amnesics and control subjects were tested on two blocks of trials, using similar stimuli and trial struc- 
tures as that used in experiment $1^{b}$ (Mitchell et al., 2000b). Memory load was equated by intermixing object and location trials. Subjects were instructed to remember both objects and locations, but only one feature, either object or location, was tested on the probe image. Because subjects could not predict whether memory for objects or locations would be tested, they were forced to maintain both features in memory. In this way, the memory load was similar to that in conjunction trials. As in experiment 1 , conjunction trials were administered in a separate block.

\section{Experiment 2}

Figure $2 b$ shows the results of our analysis. A repeated-measures ANOVA with condition, delay, and group as factors found a main effect of condition $\left(F_{(1,13)}=17.97 ; p=0.001\right)$, with lower overall accuracy when conjunctions had to be remembered $(M=80 \%)$ compared with features $(M=95 \%)$. A longer delay interval led to overall worse performance $\left(F_{(1,13)}=24.60, p=0.001\right)$. The difference between groups was marginally significant $\left(F_{(1,13)}=\right.$ $4.22, p=0.061$ ) because of the somewhat lower overall accuracy of amnesics. The interaction of condition by delay was significant $\left(F_{(1,13)}=10.13 ; p=0.007\right)$. Of interest, the interaction of condition by group was significant $\left(F_{(1,13)}=4.6 ; p=0.051\right)$. This was because of similar levels of performance by amnesics and controls in the feature condition ( $M=92$ vs $97 \%)$, but lower performance by amnesics in the conjunction condition $(M=70$ vs $90 \%$ ). Unlike experiment 1 , the interaction of condition by group by delay was not significant $(F<1)$.

Despite the fact that the memory load was similar in the feature and in the conjunction condition, amnesic patients showed a selective deficit in the conjunction condition. These findings rule out a load interpretation of amnesics' impairment and provide further support for the hypothesis that the hippocampus is important for relational processing over brief delays.

To assess whether binding deficits found in experiment 2 were caused by hippocampal or extrahippocampal damage in the MTL, we compared the performance of the H group $(n=2)$ to that of the $\mathrm{H}+$ group $(n=4)$ on the conjunction trials. No difference was found between groups $(F<1)$ and the interaction of group with delay was not significant $\left(F_{(1,4)}=1.35, p=0.31\right)$. Post hoc analyses show that the groups did not differ at the $1 \mathrm{~s}$ delay $(\mathrm{H}+$ group: $M=75 \%, \mathrm{SE}=11 \%$; $\mathrm{H}$ group: $M=83 \%$, $\mathrm{SE}=17 \% ; p=0.70)$ or the 8 s delay $(\mathrm{H}+$ group: $M=56 \%, \mathrm{SE}=$ $21 \%$; $\mathrm{H}$ group: $M=45 \%, \mathrm{SE}=33 \% ; p=0.70)$. These findings suggest that the binding deficits observed in amnesic patients can be attributed to hippocampal damage.

\section{Discussion}

The dissociation between long and short-term forms of memory in medial temporal lobe amnesia has been used to provide the linchpin evidence for the psychological distinction between short-term or working memory and long-term memory (Wickelgren, 1968; Baddeley and Warrington, 1970; Cave and Squire, 1992). Here, we show that the MTL is important for at least one type of WM: memory for relationships or conjunctions. The results of experiment 1 showed that amnesics have intact WMs for single features at delays of up to $8 \mathrm{~s}$. The fact that feature memory, memory for locations or objects, did not differ between amnesic and control groups allowed us to assess whether amnesic patients had a disproportionate deficit in relational memory. Indeed,

${ }^{b}$ Unlike Experiment 1, in Experiment 2, after the ISI, a phrase appeared on the screen for $1000 \mathrm{~ms}$ that prompted what needed to be recollected on the probe image. The phrase was "remember object," "remember location," or "remember object plus location." when required to remember an object in a location over an $8 \mathrm{~s}$ delay period, their memory was dramatically impaired. Additional analyses revealed that this deficit was closely linked to damage in the hippocampus per se and not surrounding structures.

Previous findings have provided evidence for the relational processing theory by showing that the MTL is necessary for conjunction memory over long delays (Cermak, 1976; Winocur and Weiskrantz, 1976; Chun and Phelps, 1999; Giovanello et al., 2003) (but see Stark and Squire, 2001). Our work extends the relational processing theory (Cohen and Eichenbaum, 1993; Eichenbaum, 1999) by showing that the MTL is necessary for relational processing at delays as short as $8 \mathrm{~s}$.

The task we used was originally developed by Mitchell et al. (2000a), who showed that the hippocampus was more highly activated in an fMRI study to object-location conjunction stimuli than to object or location feature stimuli. Conjunction activations were greater in young adults than older adults, and memory performance for these same stimuli was higher in young adults as compared with older adults. It was inferred from this data that the conjunction memory deficit seen in older adults was the result of subtle hippocampal atrophy. But frontal dysfunction, which is commonly seen in older adults, could not be ruled out. Also, it is was not clear from this data whether the hippocampal activation to conjunction stimuli reflected the maintenance of memory representations in working memory, or the establishment of longterm memory traces.

Like Mitchell et al. (2000a), we found that normal older adults were somewhat worse at remembering conjunctions. Normal older adults may find it more difficult to remember conjunctions because physiological factors such as age-related cell loss (Coleman and Flood, 1987) or age-related electrophysiological rigidity (Wilson et al., 2004), could serve to reduce the efficiency of information processing in the hippocampus. A more extreme case of this is observed in patients with MTL amnesia who have radical loss of hippocampal volume and a commensurate extreme loss in the ability to remember conjunctions.

\section{Elapsed time and the hippocampus}

When hippocampally based WM deficits have been observed in the past, the data collide with the paradigmatic view of memory and the hippocampus: that it is only important for LTM. For instance, two previous studies found differences between amnesic and normal control memory performance at short delays (Buffalo et al., 1998; Ryan and Cohen, 2004a). These results were interpreted as being attributable to the influence of impaired LTM in amnesia. Unfortunately, the circular logic of this interpretation (e.g., if amnesia is defined as an impairment in LTM then any memory impairment, regardless of delay, must be an impairment in LTM) renders it impotent. This problem can be skirted by defining the theoretical construct of WM as the activated contents of long-term memory (Cowan, 1995; Ericsson and Kintsch, 1995) and the functionality of WM as maintaining information from LTM in a readily accessible state (Ryan and Cohen, 2004a). However, a strong version of this definition renders WM tantamount to consciousness (O'Reilly et al., 1999) or attention, and fails to account for the ability to retain novel information in WM.

It is still unclear as to how short of a delay can elicit an impairment in amnesics. Ryan and Cohen (2004b) found that amnesic patients failed to exhibit a specific eye-movement effect (fewer fixations on previously viewed items as compared with new items) in a scene change-detection task when the delay was long, but when the delay was shortened to a little over one second, the 
performance of amnesics and controls did not differ. Our findings do little to enlighten this issue because in experiment 1 , binding deficits were found only at the $8 \mathrm{~s}$ delay, whereas in experiment 2 , binding deficits were observed across delays. In other experiments, we have observed visual WM deficits in MTL amnesics at $4 \mathrm{~s}$ delays (Olson et al., 2006). A meta-analysis of monkey and patient studies found little evidence for the existence of delay-related hippocampal-memory impairments over delays ranging from 0 to $600 \mathrm{~s}$ (Ringo, 1991). Recent neurophysiology and neuroimaging studies both support (Zola-Morgan and Squire, 1985; Murray and Mishkin, 1986; Suzuki et al., 1997; Stern et al., 2001; Ranganath and D'Esposito, 2005) and refute (Cohen et al., 1997; Zarahn et al., 2004) this assertion. In particular, some neuroimaging studies have reported hippocampal activations during WM delays (for review, see Ranganath and D'Esposito, 2005), whereas other investigators have used neuroimaging findings to argue against the hypothesis that the hippocampus is involved in WM (Zarahn et al., 2004). We speculate that elapsed time is a poor variable for predicting the inception or degree of amnesic deficits and that other factors, such as the underlying representation needed to perform the task, will more fruitfully explain amnesic deficits.

\section{Explaining relational processing deficits at short delays}

The results of experiment 2 rule out a memory-load explanation of amnesics' impairment in conjunction memory at short delays, because amnesics and controls performed no differently on feature trials, even when the memory load was increased to be similar to that on conjunction trials. Thus, amnesics' impairment was not simply a function of the number of features to be encoded, but was related specifically to the need to bind different features.

One concern that may be raised relates to the fact that performance on feature trials at the short delay was near ceiling, limiting our ability to see potential performance differences between amnesic patients and controls. However, in experiment 1, performance on feature trials was off ceiling at $8 \mathrm{~s}$ and yet no difference was found between the performance of amnesics and controls, weakening the credibility of this explanation.

The relational processing theory of the MTL offers little insight into the stage of memory processing that is reliant on such processing. One possibility is that the observed binding deficits were caused by impoverished encoding of conjunctions, leading to a fragile memory representation that quickly disintegrated. The hippocampus literature provides no support for this idea. A second possibility is that the observed binding deficits were caused by problems in maintaining relational information over time (Ryan and Cohen, 2004b). It is possible that single features, such as objects or locations can be maintained by perceptual systems in inferior temporal and superior parietal lobes (Druzgal and D'Esposito, 2001), whereas object-location conjunctions rely on the MTL for accurate maintenance. Support for this explanation is provided by fMRI studies showing sustained activity in the hippocampus over WM delays (for review, see Ranganath and D’Esposito, 2005).

\section{Conclusions}

In sum, these findings extend the relational processing theory by showing that the hippocampus is important for remembering the relationship between different features of a memory trace at short delay intervals. The data presented here suggest that the distinction between short- and long-term memory may be less impor- tant than the distinction between feature and conjunction memory in defining the role of the hippocampus in episodic memory.

\section{References}

Baddeley A (2003) Working memory: looking back and looking forward. Nat Rev Neurosci 4:829-839.

Buffalo EA, Reber PJ, Squire LR (1998) The human perirhinal cortex and recognition memory. Hippocampus 8:330-339.

Cave CB, Squire L (1992) Intact verbal and nonverbal short-term memory following damage to the human hippocampus. Hippocampus 2:151-163.

Cermak L (1976) The encoding capacity of a patient with amnesia due to encephalitis. Neuropsychologia 14:311-326.

Chun MM, Phelps EA (1999) Memory deficits for implicit contextual information in amnesic subjects with hippocampal damage. Nat Neurosci 2:844-847.

Cohen JD, Perlstein WM, Braver TS, Nystrom LE, Noll DC, Jonides J, Smith EE (1997) Temporal dynamics of brain activation during a working memory task. Nature 386:604-608.

Cohen NJ, Eichenbaum H (1993) Memory, amnesia, and the hippocampal system. Cambridge, MA: MIT.

Coleman PD, Flood DG (1987) Neuron numbers and dendritic extent in normal aging and Alzheimer's disease. Neurobiol Aging 8:521-545.

Cowan N (1995) Attention and memory: an integrated framework. New York: Oxford UP.

Davachi L, Mitchell J, Wagner AD (2003) Multiple routes to memory: distinct medial temporal lobe processes build up item and source memories. Proc Natl Acad Sci USA 100:2157-2162.

Druzgal TJ, D'Esposito MD (2001) A neural network reflecting decisions about human faces. Neuron 32:947-955.

Eichenbaum H (1999) The hippocampus and mechanisms of declarative memory. Behav Brain Res 103:123-133.

Ericsson KA, Kintsch W (1995) Long-term working memory. Psychol Rev 102:211-245.

Giovanello KS, Verfaellie M, Keane MM (2003) Disproportionate deficit in associative recognition relative to item recognition in global amnesia. Cogn Affect Behav Neurosci 3:186-194.

Giovanello KS, Schnyer DM, Verfaellie M (2004) A critical role for the anterior hippocampus in relational memory: evidence from an fMRI study comparing associative and item recognition. Hippocampus 14:5-8.

Henke K, Buck A, Weber B, Wieser HG (1997) Human hippocampus establishes associations in memory. Hippocampus 7:249-256.

Hirsh R (1974) The hippocampus and contextual retrieval of information from memory: a theory. Behav Biol 12:421-444.

Kirwan CB, Stark CEL (2004) Medial temporal lobe activation during encoding and retrieval of novel face-name pairs. Hippocampus 14:919-930.

Mitchell KJ, Johnson MK, Raye CL, D’Esposito M (2000a) fMRI evidence of age-related hippocampal dysfunction in feature binding in working memory. Brain Res Cogn Brain Res 10:197-206.

Mitchell KJ, Johnson MK, Raye CL, Mather M, D’Esposito M (2000b) Aging and reflective processes of working memory: binding and test load deficits. Psychol Aging 15:527-541.

Murray EA, Mishkin M (1986) Visual recognition in monkeys following rhinal cortical ablations combined with either amygdalectomy or hippocampectomy. J Neurosci 6:1991-2003.

Olson IR, Moore KS, Stark M, Chatterjee A (2006) Visual working memory is impaired when the medial temporal lobe is damaged. J Cogn Neurosci, in press.

O’Reilly RC, Braver TS, Cohen JD (1999) A biologically based computational model of working memory. In: Models of working memory: mechanisms of active maintenance and executive control (Miyake A, Shah P, eds), pp 375-411. Cambridge, UK: Cambridge UP.

Ranganath C, D'Esposito M (2005) Directing the mind's eye: prefrontal, inferior, and medial temporal mechanisms for visual working memory. Curr Opin Neurobiol 15:175-182.

Ranganath C, Yonelinas AP, Cohen MX, Dy CJ, Tom SM, D'Esposito MD (2004) Dissociable correlates of recollection and familiarity within the medial temporal lobes. Neuropsychologia 42:2-13.

Ringo JL (1991) Memory decays at the same rate in macaques with and without brain lesions when expressed in d' or arcsine terms. Behav Brain Res 42:123-134.

Rossion B, Pourtois G (2004) Revisiting Snodgrass and Vanderwart's object 
set: the role of surface detail in basic-level object recognition. Perception 33:217-236

Ryan JD, Cohen NJ (2004a) The nature of change detection and online representations of scenes. J Exp Psychol Hum Percept Perform 30:988-1015.

Ryan JD, Cohen NJ (2004b) Processing and short-term retention of relational information in amnesia. Neuropsychologia 42:497-511.

Scoville WB, Milner B (1957) Loss of recent memory after bilateral hippocampal lesions. J Neuropsychiatry Clin Neurosci 20:11-21.

Stark CEL, Squire LR (2001) Simple and associative recognition memory in the hippocampal region. Learn Mem 8:190-197.

Stern CE, Sherman SJ, Kirchhoff BA, Hasselmo ME (2001) Medial temporal and prefrontal contributions to working memory tasks with novel and familiar stimuli. Hippocampus 11:337-346.

Sutherland RJ, Rudy JW (1989) Configural association theory: the role of the hippocampal formation in learning, memory, and amnesia. Psychobiology 17:129-144.

Suzuki WA, Miller EK, Desimone R (1997) Object and place memory in the macaque entorhinal cortex. J Neurophysiol 78:1062-1081.

Turriziani P, Fadda L, Caltagirone C, Carlesimo GA (2004) Recognition memory for single items and for associations in amnesic patients. Neuropsychologia 42:426-433.
Wechsler D (1997a) Wechsler adult intelligence scale, Ed 3: Administration and scoring manual. San Antonio, TX: Psychological Corporation.

Wechsler D (1997b) Wechsler memory scale, Ed 3: Administration and scoring manual. San Antonio, TX: Psychological Corporation.

Wickelgren WA (1968) Sparing of short-term memory in an amnesic patient: implications for strength theory of memory. Neuropsychologia 6:235-244.

Wilson IA, Ikonen S, Gurevicience I, McMahan RW, Gallagher M, Eichenbaum H, Tanila H (2004) Cognitive aging and the hippocampus: how old rats represent new environments. J Neurosci 24:3870-3878.

Winocur G, Weiskrantz L (1976) An investigation of paired-associate learning in amnesia. Neuropsychologia 14:97-110.

Yonelinas AP, Hopfinger JB, Buonocore MH, Kroll NE, Baynes K (2001) Hippocampal, parahippocampal and occipital-temporal contributions to associative and item recognition memory: an fMRI study. NeuroReport 12:359-363.

Zarahn E, Rakitin B, Abela D, Flynn J, Stern Y (2004) Positive evidence against human hippocampal involvement in working memory maintenance of familiar stimuli. Cereb Cortex 15:303-316.

Zola-Morgan S, Squire LR (1985) Medial temporal lesions on monkeys impair memory in a variety of tasks sensitive to human amnesia. Behav Neurosci 99:22-34. 